\title{
High-Quality 2D Metal-Organic Coordination Network Providing Giant Cavities within Mesoscale Domains
}

\author{
Dirk Kühne, ${ }^{\dagger}$ Florian Klappenberger, ${ }^{*, \dagger}$ Régis Decker, ${ }^{\ddagger}$ Uta Schlickum, ${ }^{\ddagger}$ Harald Brune, ${ }^{\ddagger}$ \\ Svetlana Klyatskaya, ${ }^{\S}$ Mario Ruben, ${ }^{*,}$ and Johannes V. Barth ${ }^{*, \dagger}$ \\ Physik Department E20, TU München, James-Franck Str, D-85748 Garching, Germany, Institut de Physique des \\ Nanostructures, EPF Lausanne, CH-1015 Lausanne, Switzerland, and Institut für Nanotechnologie,
} Forschungszentrum Karlsruhe, D-76021 Karlsruhe, Germany

Received December 28, 2008; E-mail: fklapp@ph.tum.de; mario.ruben@int.fzk.de; jvb@ph.tum.de

A particular important objective in the field of coordination polymers and metal-organic frameworks is the realization of porous structures providing spaces with a well-defined geometry and chemical environment. ${ }^{1,2}$ However, in three dimensions, mainly due to interpenetration of the forming networks, it is challenging to achieve open spaces reaching the mesoporous regime, i.e., 20-500 $\AA$, based on building principles rooted in coordination interactions or supramolecular engineering. ${ }^{2}$ Porous molecular layers can also be stabilized by the presence of surfaces, where they represent versatile functional nanoarchitectures. Thus over the past years a series of bottom-up fabrication schemes has been developed to realize two-dimensional (2D) open molecular networks, frequently with limited domain size, using hydrogen-bonding, ${ }^{3}$ metal-directed assembly, ${ }^{4}$ the organization of flexible species, ${ }^{5}$ or covalent chemical reactions. ${ }^{6}$

Similar to the situation encountered with 3D systems, the engineering of extended pore sizes organized in mesoscopically regular single-phase domains is particularly demanding. A recent step forward in this direction was an anthraquinone honeycomb network achieved on a $\mathrm{Cu}(111)$ surface, with a rather large pore diameter of $\sim 50 \AA{ }^{7}$ Because its formation relies on the interference of substrate-mediated long-range interactions, which are rather weak and hard to control in a systematic manner, ${ }^{8}$ it turned out to be stable only up to $200 \mathrm{~K}$. On the other hand, using only easy-tocontrol parameters we developed an approach to fabricate open and robust metal-organic nanomeshes on the $\operatorname{Ag}(111)$ surface that allows tuning the cavity size in a straightforward fashion using dicarbonitrile-polyphenyl linkers with variable length $\left(\mathrm{CN}-\mathrm{Ph}_{n}-\mathrm{CN}\right.$, whereby $n$ is 3,4 , or 5$)$ coordinated by cobalt centers. ${ }^{9}$ However, the longest linkers produced networks of reduced quality and deviations from the threefold symmetry of the coordination node appeared therewith.

Herein we report a scanning tunneling microscopy (STM) investigation of a highly regular $2 \mathrm{D}$ nanoporous metal-organic network with a large pore diameter of $\sim 67 \AA$. To this end we employed a de novo synthesized para-hexaphenyl-dicarbonitrile linker 1 (depicted in Scheme 1) with a length of $29.6 \AA$ (as calculated in the semiempirical AM1 framework). The resulting large $29 \mathrm{~nm}^{2}$ cell size (corresponding to an $\sim 24 \mathrm{~nm}^{2}$ van der Waals cavity), expressed in the Co-directed assembly of nanomeshes, allowed for atomic resolution imaging of the atomic silver lattice therein. Thus we can rationalize the high quality of the fabricated networks in terms of their epitaxial fit where coordination nodes reside preferentially at hollow sites and the linkers strictly follow

\footnotetext{
TU München.

EPF Lausanne.

$\$$ Forschungszentrum Karlsruhe.
}

Scheme 1. Molecular Linker para-Hexaphenyl-dicarbonitrile; $C(H$, N) Atoms in Gray (White, Blue)

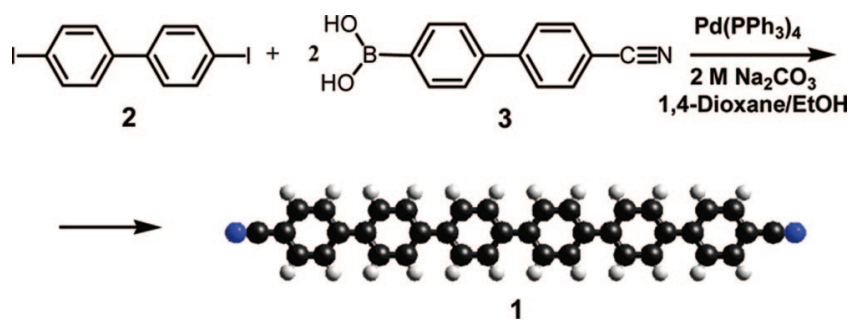

high-symmetry crystallographic orientations on the employed $\operatorname{Ag}(111)$ substrate.

The synthesis of the rod-like $\mathrm{NC}-\mathrm{Ph}_{6}-\mathrm{CN}$ (1) molecule was developed on the basis of Suzuki coupling of 4,4'-bis-iodo-diphenyl 2 with 2 equiv of boronic acid $\mathbf{3}$ in the presence of catalytic amounts of $\operatorname{Pd}(0)$ (see the Supporting Information; for related work with shorter species, see ref 10). The experiments were performed using a home-built ultrahigh vacuum (base pressure $3 \times 10^{-11} \mathrm{mbar}$ ) lowtemperature STM instrument. ${ }^{11}$ The $\mathrm{Ag}(111)$ substrate was prepared by cycles of $\mathrm{Ar}^{+}$sputtering and annealing to $740 \mathrm{~K}$ to obtain flat terraces separated by monatomic steps. Linkers were deposited from a quartz crucible in an organic molecular beam epitaxy source at $572 \mathrm{~K}$, with the substrate kept at $300 \mathrm{~K}$. Molecular films were subsequently exposed to a beam of Co atoms to assemble metal-organic networks. Following preparation the sample was cooled down and STM data were acquired at $T=8$ and 77-300 $\mathrm{K}$, respectively.

An STM overview image is reproduced in Figure 1a. The data reveal a highly regular nanomesh dividing the surface into equal honeycomb-shaped units. The formation of the network is attributed to threefold coordination of carbonitrile groups to one Co atom, in analogy to our previous experimental and theoretical work. ${ }^{9}$ The regularity indicates that there must be an effective self-correction during the metallosupramolecular assembly, efficiently eliminating possible transient structural defects. The sole spurious features in the supramolecular organization, which however do not perturb the honeycomb order, are a number of linker molecules trapped in the cavities during cooling of the sample. The amount of the trapped molecules depends on the stoichiometric ratio between metal centers and linkers and can be reduced by careful fine-tuning of the deposition parameters.

The image in Figure 1b shows a close-up view of several individual nanopores, each of which provides the same $23.7 \mathrm{~nm}^{2}$ hexagonal empty space. Molecules appear as $1.7 \AA$ protrusions. Co centers at mesh nodes (apparent height $1.2 \AA$ ) are resolved here, in contrast to earlier observations. ${ }^{9}$ 
a)

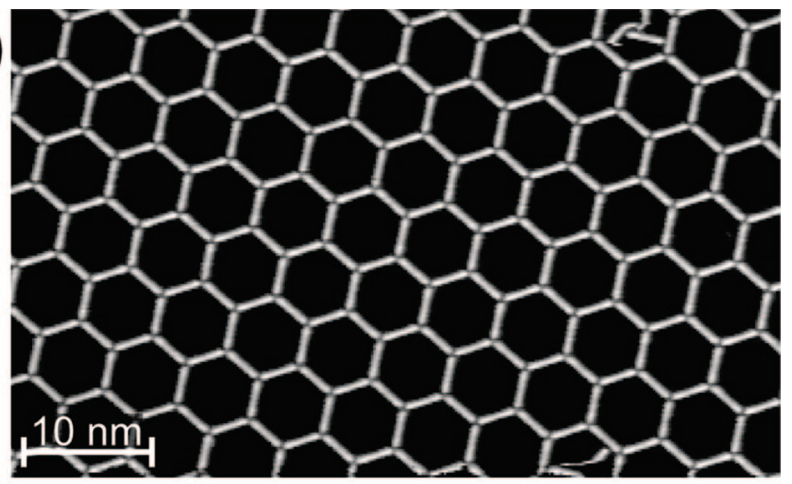

b)

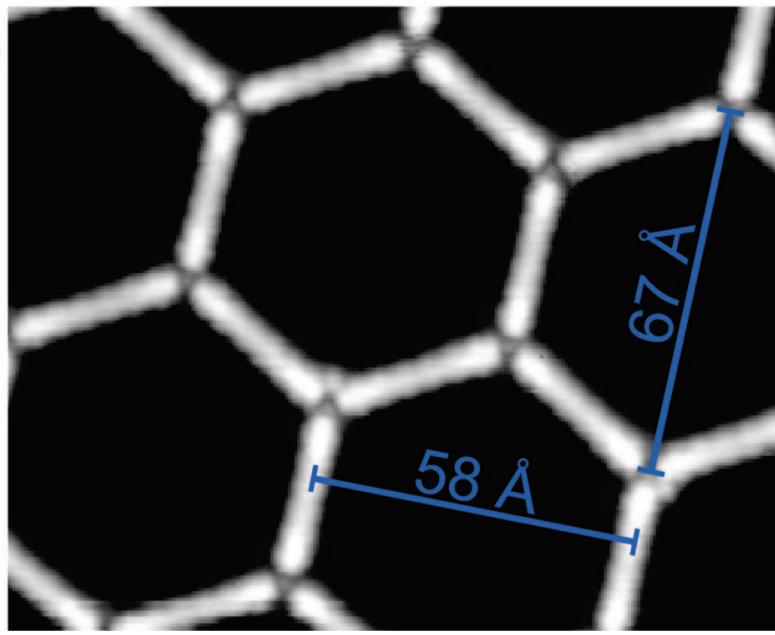

Figure 1. (a) Overview STM topographic image of the metal-organic nanomesh formed by Co-directed assembly of $\mathrm{NC}-\mathrm{Ph}_{6}-\mathrm{CN}$ linkers on $\mathrm{Ag}(111)$. In some cavities a number of linkers are trapped [the sample bias voltage was $V_{\mathrm{B}}=0.5 \mathrm{~V}$, and the tunneling current was $I_{\mathrm{T}}=0.1 \mathrm{nA}$. (b) High regularity of the superlattice featuring a $67 \AA$ pore diameter $\left[V_{\mathrm{B}}=\right.$ $\left.0.5 \mathrm{~V}, I_{\mathrm{T}}=0.1 \mathrm{nA}\right]$.

The high regularity of the Co-hexaphenyl-dicarbonitrile nanomesh suggests that it is stabilized by the underlying first atomic $\mathrm{Ag}(111)$ plane. To precisely assess the effect of the epitaxial fit with respect to the substrate we performed high-resolution STM measurements simultaneously resolving molecular linkers and atomic corrugation. Analysis of the obtained images unambiguously determines the relative orientation and distances of the surface lattice and nanomesh and confirms commensurability of the superstructure. The exemplary data in Figure 2 (left) show the positions of individual silver atoms in the cavities together with the molecules appearing as depressions (black). The image was recorded with standard tunneling parameters; thus the special resolution properties are most likely related to a special state of the tip, equally indicated by the atypical and contrast inverted molecular appearance. Our analysis leads to the structure model presented in the right part of Figure 2 partially superimposed to the experimental data in the depicted image's central part. The linker orientations are parallel to the surface following strictly the $\langle 11-2\rangle$ surface crystallographic directions, and Co atoms occupy both of the nonequivalent threefold hollow sites (fcc and hcp, respectively).

With the present metal-organic network a further decisive factor is the fit of the coordination motif and bond length on the substrate. Because the linker's electronic structure is only weakly affected by the subjacent silver lattice, ${ }^{9,11}$ one can assume that its length is close to that of isolated species. The model represented in Figure 2 takes this into account and allows us to estimate a $\mathrm{Co}-\mathrm{NC}$ bond distance of $\sim 1.9 \AA$, similar to values found for nanomeshes with

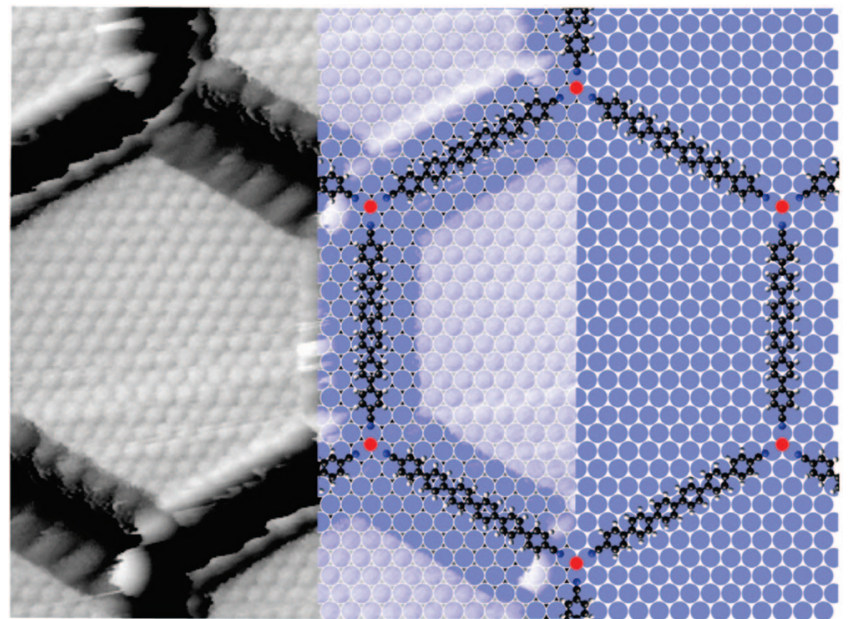

Figure 2. Atomically resolved $\mathrm{Ag}(111)$ surface lattice within the nanocavities of the Co-hexaphenyl-dicarbonitrile nanomesh [left; $\left.V_{\mathrm{B}}=0.6 \mathrm{~V}, I_{\mathrm{T}}=0.1 \mathrm{nA}\right]$. A corresponding ball model is superposed on the tunneling image in the center. The molecular backbones are oriented in registry along surface crystallographic $\langle 11-2\rangle$ directions with the coordination nodes residing at substrate hollow sites. The ball model at the right visualizes the perfect registry between network and substrate [Ag, Co atoms in blue, red; linkers as in Scheme 1]. Nearest-neighbor distance of $\mathrm{Ag}$ atoms is $2.889 \AA$

shorter linkers ${ }^{9}$ and related $3 \mathrm{D}$ compounds. ${ }^{12}$ The size of the superstructure unit cell is determined to be $(20 \times 20)$ substrate lattice unit vectors.

For comparison, in the orientational ordering of pure parahexaphenyl-dicarbonitrile superstructures on $\operatorname{Ag}(111)$, we found that the molecules line up exclusively in $\langle 1-10\rangle$ or $\langle 11-2\rangle$ directions, forming a variety of distinct molecular superlattices. The linkers' preference for these two crystallographic directions is also reflected in the metal-organic ordering: In addition to the orientation along the $\langle 11-2\rangle$ direction as in the nanomesh arrangement described above, we identified minority domains (with a fractional coverage of $\sim 15 \%$ of the nanomesh area) that are equally well ordered but with the molecules oriented along $\langle 1-10\rangle$ directions, i.e., a superlattice rotated by $30^{\circ}$ comprising a $40 \times 40$ unit cell. The increased unit cell results from the fact that Co atoms partially occupy both bridge and on top sites (note that the bonding energy variation for isolated Co adatoms is quite small ${ }^{14}$ and presumably further reduced upon lateral coordination). In the minority domains, linkers are aligned along the close-packed direction and have an identical orientation and surface registry as individual parahexaphenyl molecules on the same substrate. ${ }^{13}$ Altogether, the Co atoms exhibit varying stacking sites in the nanomesh domains, whereas the linker orientations are unaffected by the presence of the metal centers. This suggests that the orientational ordering of the metal-organic network is dominated by the linkers' hexaphenyl backbone interaction with the surface.

Analysis of large-scale images shows that domain sizes are generally limited only by the step-terrace topology. Even terraces exceeding $700 \mathrm{~nm}$ in width are covered with defect-free single domains (see Figure 3a and the magnified image shown in Figure S3 in the Supporting Information), whereby the high regularity of the nanomesh is preserved over the entire substrate terrace; i.e., the threefold symmetry of the coordination nodes is perfectly obeyed area-wide. We conclude that due to the error correction during metal-directed assembly and the commensurability of the superstructure the domain size limit inherent in the metallosupramolecular organization is well beyond the $\mu \mathrm{m}$ range. Because the assembly requires essentially a balance between coordination interactions and energy barriers for rotational and translation motions ensuring 

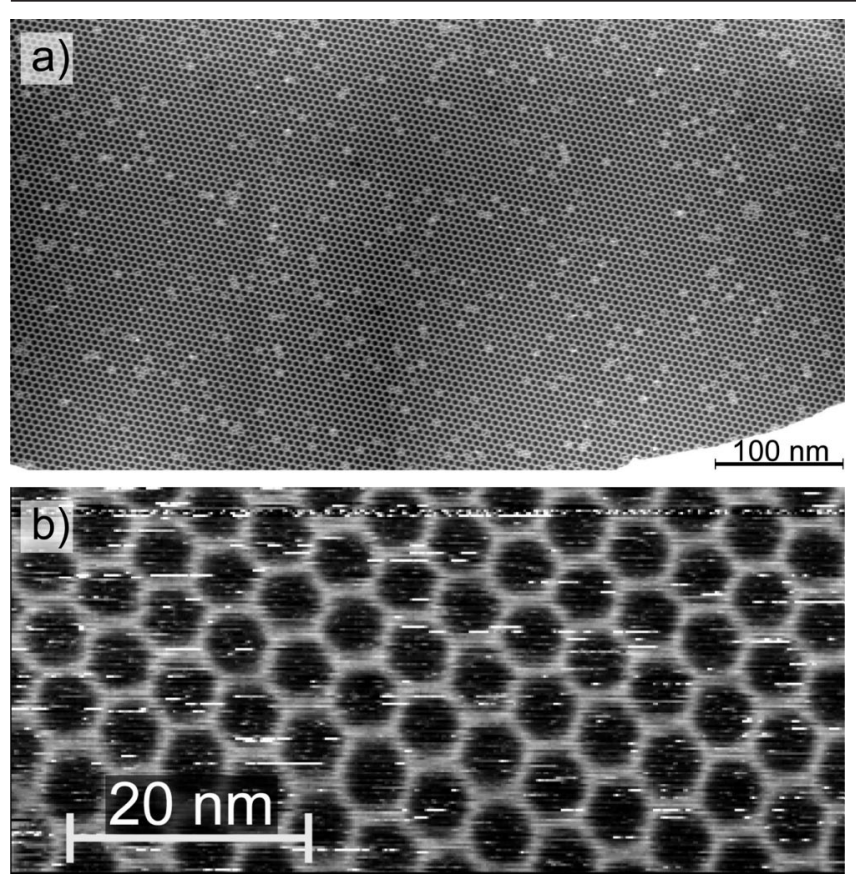

Figure 3. (a) Large-scale STM image of the metal-organic nanomesh showing its regularity over a $240000 \mathrm{~nm}^{2}$ surface area $\left[V_{\mathrm{B}}=1 \mathrm{~V}, I_{\mathrm{T}}=0.1\right.$ nA]. (b) Nanomesh stability demonstrated by room temperature STM measurement. White dashes appearing in scan lines indicate that the molecules trapped at low temperatures in the voids diffuse now freely across the rims $\left[V_{\mathrm{B}}=0.34 \mathrm{~V}, I_{\mathrm{T}}=26 \mathrm{pA}\right]$.

surface mass transport and self-correction, ${ }^{8}$ our methodology is expected to be extendable to $2 \mathrm{D}$ nanomeshes with even larger pore sizes.

The robustness of the metal-organic network was tested by a series of STM measurements in the 77-300 K range. The nanomesh proved stable not only against thermal excitations up to room temperature but also against the additional influence of the tunneling current and electrical field caused by the tip scanning (see the image in Figure 3b; a larger scale image is shown in Figure S1 in the Supporting Information; note that narrower meshes qualify even as templates to steer metal cluster growth ${ }^{15}$ ). In a data set recorded at $195 \mathrm{~K}$ both empty and occupied pores were clearly distinguishable, whereas between subsequent images ( $\sim 2$ min time lapse) guest molecules changed their host pores. Thus we conclude that at room temperature uncoordinated linkers are highly mobile and rapidly migrate across the network rims. Accordingly, they are elusive to STM imaging, appearing as dashes in the individual scan lines (see Figure $3 b$ ).

In conclusion, we demonstrated the fabrication of a robust open nanomesh on a noble metal surface using cobalt-directed assembly of para-hexaphenyl-dicarbonitrile linker molecules. The well- defined metal-organic superlattice with $24 \mathrm{~nm}^{2}$ cavities fits precisely onto the substrate atomic lattice. The large pore size and perfect regularity opens novel avenues to steer the organization of matter at the nanoscale, to explore 2D host-guest systems as well as the confinement of molecular rotating units and reactive species, or to engineer arrays of quantum corrals.

Acknowledgment. This work was supported by the European Science Foundation Collaborative Research Programme FunSMARTs.

Supporting Information Available: Detailed description of the synthesis of compound 1. Additional large-scale images and room temperature data. This material is available free of charge via the Internet at http://pubs.acs.org.

\section{References}

(1) Yaghi, O. M.; O'Keeffe, M.; Ockwig, N. W.; Chae, H. K.; Eddaoudi, M.; Kim, J. Nature 2003, 423, 705.

(2) Kitagawa, S.; Kitaura, R.; Noro, S. Angew. Chem. 2004, 43, 2334.

(3) Griessl, S.; Lackinger, M.; Edelwirth, M.; Hietschold, M.; Heckl, W. M. Single Molecules 2002, 3, 25. Dmitriev, A.; Lin, N.; Weckesser, J.; Barth, J. V.; Kern, K. J. Phys. Chem. B 2002, 106, 6907. Theobald, J. A.; Oxtoby, N. S.; Phillips, M. A.; Champness, N. R.; Beton, P. H. Nature 2003, 424, 1029. Stöhr, M.; Wahl, M.; Galka, C. H.; Riehm, T.; Jung, T. A.; Gade, L. H. Angew. Chem. 2005, 44, 7394. Ruben, M.; Payer, D.; Comisso, A.; Gattinoni, C.; Lin, N.; Collin, J.-P.; Sauvage, J.-P.; De Vita, A.; Kern, K. J. Am. Chem. Soc. 2006, 128, 15644. Madueno, R.; Räisänen, M. T.; Silien, C.; Buck, M. Nature 2008, 454, 618.

(4) Dmitriev, A.; Spillmann, H.; Lin, N.; Barth, J. V.; Kern, K. Angew. Chem. 2003, 41, 2670. Stepanow, S.; Lingenfelder, M.; Dmitriev, A.; Spillmann, H.; Delvigne, E.; Lin, N.; Deng, X.; Cai, C.; Barth, J. V.; Kern, K. Nat. Mater. 2004, 3, 229. Spillmann, H.; Dmitriev, A.; Lin, N.; Messina, P.; Barth, J. V.; Kern, K. J. Am. Chem. Soc. 2003, 125, 10725. Stepanow, S.; Lin, N.; Barth, J. V. J. Phys. Condens. Matter 2008, 20, 184002. Stepanow, S.; Lin, N.; Barth, J. V.; Kern, K. J. Phys. Chem. B 2006, 110, 23472.

(5) Schull, G.; Douillard, L.; Fiorini-Debuisschert, C.; Charra, F.; Mathevet, F.; Kreher, D.; Attias, A.-J. Nano Lett. 2006, 6, 1360. Spillmann, H.; Kiebele, A.; Jung, T. A.; Bonifazi, D.; Cheng, F.; Diederich, F. Adv. Mater. 2006, 18, 275. Mena-Osteritz, E.; Bäuerle, P. Adv. Mater. 2006, 18, 447. Tahara, K.; Furukawa, S.; Uji-i, H.; Uchino, T.; Ichikawa, T.; Zhang, J.; Mamdouh, W.; Sonoda, M.; De Schryver, F. C.; De Feyter, S.; Tobe, Y. J. Am. Chem. Soc. 2006, 128, 16613.

(6) Zwaneveld, N. A. A.; Pawlak, R.; Abel, M.; Catalin, D.; Gigmes, D.; Bertin, D.; Porte, L. J. Am. Chem. Soc. 2008, 130, 6678. Weigelt, S.; Bombis, C.; Busse, C.; Knudsen, M. M.; Gothelf, K. V.; Lœgsgaard, E.; Besenbacher, F.; Linderoth, T. R. ACS Nano 2008, 2, 651.

(7) Pawin, G.; Wong, K. L.; Kwon, K.-Y.; Bartels, L. Science 2006, 313, 961.

(8) Barth, J. V. Annu. Rev. Phys. Chem. 2007, 58, 375.

(9) Schlickum, U.; Decker, R.; Klappenberger, F.; Zoppellaro, G.; Klyatskaya, S.; Ruben, M.; Silanes, I.; Arnau, A.; Kern, K.; Brune, H.; Barth, J. V. Nano Lett. 2007, 7, 3813 .

(10) Schlickum, U.; Decker, R.; Klappenberger, F.; Zoppellaro, G.; Klyatskaya, S.; Auwärter, W.; Neppl, S.; Kern, K.; Brune, H.; Ruben, M.; Barth, J. V. J. Am. Chem. Soc. 2008, 130, 11778.

(11) Clair, S. PhD thesis, EPF Lausanne (2004). Clair, S.; Pons, S.; Seitsonen, A. P.; Brune, H.; Kern, K.; Barth, J. V. J. Phys. Chem. B 2004, 108, 19392.

(12) Przychodzen, P.; Korzeniak, T.; Podgajny, R.; Sieklucka, B. Coord. Chem. Rev. 2006, 250, 2234.

(13) Braun, K.-F.; Hla, S.-W. Nano Lett. 2005, 5, 73.

(14) Schiffrin, A.; Reichert, J.; Auwärter, W.; Jahnz, G.; Pennec, Y.; WeberBargioni, A.; Stepanyuk, V. S.; Niebergall, L.; Bruno, P.; Barth, J. V. Phys. Rev. B 2008, 78, 035424 .

(15) Decker, R.; Schlickum, U.; Klappenberger, F.; Zoppellaro, G.; Klyatskaya, S.; Ruben, M.; Barth, J. V.; Brune, H. Appl. Phys. Lett. 2008, 93, 243102.

JA809946Z 